\title{
Gastroenteropancreatic neuroendocrine tumors: current perspectives and future challenges
}

\author{
Rocio Garcia-Carbonero
}

Published online: 20 December 2013

(C) Springer Science+Business Media New York 2013

Gastroenteropancreatic neuroendocrine tumors (GEP-NETs) comprise a heterogeneous family of diseases of increasing incidence and challenging clinical management. Although traditionally considered indolent tumors, their clinical behavior is often unpredictable and can sometimes be very aggressive. The wide range of classifications proposed for these tumors based on their embryological or anatomical origin, morphological and histological features, biochemical profile, and/or clinical behavior clearly illustrates their high complexity and diverse nature.

The incidence of NETs in the Caucasian population has substantially increased over the past three decades from 1.09 (1973) to 5.25 (2004) new cases per 100,000 inhabitants annually. About two thirds of NETs are of gastrointestinal or pancreatic origin (GEP-NETs) and, among these, the most common site of origin is the small intestine [1]. Although GEP-NETs represent only $2 \%$ of all gastrointestinal tumors, the fact that they have a better prognosis than exocrine cancers makes their relative prevalence much higher, being the second most prevalent gastrointestinal malignancy after colorectal adenocarcinoma. These tumors are diagnosed at a younger age than carcinomas, usually during the fifth decade of life. About one third is so-called functioning tumors, a unique feature of NETs that is the consequence of excess hormone production by the tumor.

According to the data from the US Surveillance, Epidemiology and End Results population registry, $27 \%$ of GEPNETs display metastatic disease at presentation (64\% pancreatic vs. $30 \%$ ileal and $50 \%$ poorly differentiated tumors $v s$.

R. Garcia-Carbonero $(\bowtie)$

Oncology Department, Hospital Universitario Virgen del Rocío Instituto de Biomedicina de Sevilla (IBIS) [Universidad de Sevilla, CSIC, HUVR], Avenida Manuel Siurot S/N, 41013 Sevilla, Spain e-mail: rgcarbonero@gmail.com
$21 \%$ well-differentiated tumors) [1]. These numbers, however, are generally far higher in European databases (44$73 \%$ ). Nevertheless, the incidence may be overestimated in our European registries as they are generally hospital rather than population-based registries with a high representation of big referral centers [2, 3]. Overall survival rates may also widely vary from 30 to $90 \%$ at 5 years, as prognosis of NETs depends upon many factors, the most important being the primary tumor site, the extent of disease, the histological grade of differentiation, and the proliferative index (ki-67 or mitotic index). The distribution of these variables in each series may therefore have a considerable influence on survival rates reported.

Surgery is the only potentially curative therapeutic strategy in localized disease and may also play a role in advanced stages. Patients with advanced disease are, however, essentially incurable. A number of therapeutic strategies, nevertheless, are useful to improve symptom control, and some of them are also able to slow tumor progression. These include somatostatin analogs, a number of liver-directed ablative techniques or chemoembolization (patients with predominantly hepatic disease), peptide receptor radionuclide therapy when available, and systemic drug therapy (somatostatin analogs, interferon, chemotherapy, and targeted agents).

In this scenario, the recently approved targeted agents sunitinib and everolimus have generated great enthusiasm in the field, as they have demonstrated for the first time in adequately designed clinical trials that there are agents able to positively modify the natural history of this disease [4]. As a consequence, an explosion of new drugs is currently being explored in the context of NETs. How to optimize drug sequencing or combination strategies is still a matter of debate. Recent major advances have also been made, unraveling the biology of this class of tumors [5]. Nevertheless, there is much room for improvement, and more efforts in basic, 
translational, and clinical research will be necessary in the following years for progress to be made. Further work shall also be pursued to develop predictive biomarkers that can help select the most appropriate patients for each particular therapy and shall therefore improve the benefit-risk balance of these new therapeutic strategies and ultimately contribute to further improve the prognosis of our patients.

The Spanish Group of Neuroendocrine Tumors (GETNE), a national multidisciplinary cooperative group for the promotion of research and continued medical education in NETs, aware of this rapidly evolving and increasingly demanding field, considered it would be of interest to the NET community to publish the contents from the "VIII GETNE International Symposium on GEP-NETs" held in Seville (Spain) in October of 2012. In this symposium, the state of the art regarding biology, diagnosis, and treatment of NETs was updated by key international leaders in the field and is now summarized in this Special Issue.

\section{References}

1. Yao, J. C., Hassan, M., Phan, A., et al. (2008). One hundred years after "carcinoid": epidemiology of and prognostic factors for neuroendocrine tumors in 35,825 cases in the United States. Journal of Clinical Oncology, 26, 3063-3072.

2. Garcia-Carbonero, R., Capdevila, J., Crespo-Herrero, G., et al. (2010). Incidence, patterns of care and prognostic factors for outcome of gastroenteropancreatic neuroendocrine tumors (GEP-NETs): results from the National Cancer Registry of Spain (RGETNE). Annals of Oncology, 21, 1794-1803.

3. Ploeckinger, U., Kloeppel, G., Wiedenmann, B., et al. (2009). The German NET-registry: an audit on the diagnosis and therapy of neuroendocrine tumors. Neuroendocrinology, 90, 349-363.

4. Benavent, M., de Miguel, M. J., \& Garcia-Carbonero, R. (2012). New targeted agents in gastroenteropancreatic neuroendocrine tumors. Targeted Oncology, 7, 99-106.

5. Jiao, Y., Shi, C., Edil, B. H., de Wilde, R. F., Klimstra, D. S., Maitra, A., Schulick, R. D., Tang, L. H., Wolfgang, C. L., Choti, M. A., Velculescu, V. E., Diaz, L. A., Jr., Vogelstein, B., Kinzler, K. W., Hruban, R. H., \& Papadopoulos, N. (2011). DAXX/ATRX, MEN1, and $\mathrm{mTOR}$ pathway genes are frequently altered in pancreatic neuroendocrine tumors. Science, 331(6021), 1199-1203. 